\title{
Measurement of Countable Compactness and Lindelöf Property in RL-Fuzzy Topological Spaces
}

\author{
Xiongwei Zhang, ${ }^{1}$ Ibtesam Alshammari $\mathbb{D}^{2},{ }^{2}$ and A. Ghareeb $\mathbb{D}^{3,4}$ \\ ${ }^{1}$ School of Mathematics and Statistics, Yulin University, Yulin 719000, China \\ ${ }^{2}$ Department of Mathematics, Faculty of Science, University of Hafr Al Batin, Hafr Al Batin 31991, Saudi Arabia \\ ${ }^{3}$ Department of Mathematics, Faculty of Science, South Valley University, Qena, Egypt \\ ${ }^{4}$ Department of Mathematics, Faculty of Science, Al-Baha University, Al-Baha 65799, Saudi Arabia \\ Correspondence should be addressed to A. Ghareeb; a.ghareeb@sci.svu.edu.eg
}

Received 28 December 2020; Revised 16 January 2021; Accepted 19 January 2021; Published 8 February 2021

Academic Editor: Ahmed Mostafa Khalil

Copyright (c) 2021 Xiongwei Zhang et al. This is an open access article distributed under the Creative Commons Attribution License, which permits unrestricted use, distribution, and reproduction in any medium, provided the original work is properly cited.

\begin{abstract}
Based on the concepts of pseudocomplement of $L$-subsets and the implication operator where $L$ is a completely distributive lattice with order-reversing involution, the definition of countable RL-fuzzy compactness degree and the Lindelöf property degree of an $L$-subset in RL-fuzzy topology are introduced and characterized. Since $L$-fuzzy topology in the sense of Kubiak and Sostak is a special case of RL-fuzzy topology, the degrees of RL-fuzzy compactness and the Lindelöf property are generalizations of the corresponding degrees in $L$-fuzzy topology.
\end{abstract}

\section{Introduction}

Combining with fuzzy set theory, Chang [1] introduced the concept of fuzzy topology together with the definition of compactness by means of open cover in 1968. Afterwards, several researchers have tried successfully to extend the theory of compactness from the crisp topology to fuzzy setting [2-8]. The disadvantage of Chang's fuzzy topology is that the open $L$-subsets were fuzzy, but the topology consisting of those open $L$-subsets is still a crisp subset of $L^{X}$. This prompted Höhle [9] to make the first attempt to fuzzifying the openness in 1980 . Later on, Höhle's attempt developed independently by Kubiak [10] and Šostak [11] to $L$-fuzzy topology in 1985. In an $L$-fuzzy topology, open $L$-subsets are not crisp subset, and topology comprising those open $L$-subsets is an $L$-subset of $L^{X}$. In the setting of $L$-fuzzy topologies, many researchers have also made plentiful investigations on the notion of compactness ([12-25]).

In [26], $\mathrm{Li}$ and $\mathrm{Li}$ introduced $L$-topology on $L$-subset and discussed some of its related properties. The new kind of topology is called an RL-topology which is a generalization of $L$-topology. Furthermore, they introduced and characterized the concepts of RL-continuous function and RL-compactness by means of an inequality. As an extension of RL-topology,
RL-fuzzy topology on an L-subset is introduced in [27]. Kubiak-Šostak's L-fuzzy topology and RL-topology are special cases of RL-fuzzy topology. The RL-fuzzy compactness of $\mathrm{RL}$-fuzzy topology is further studied. They proved that the union of two RL-fuzzy compact $L$-subsets is also RL-fuzzy compact, and the intersection of an RL-fuzzy compact $L$-subset and RL-closed subset is also RL-fuzzy compact. Moreover, they showed that RL-fuzzy compactness is an invariant under RL-fuzzy continuous functions.

In this study, we introduce and characterize the degree of countable RL-fuzzy compactness and the RL-Lindelöf property of an $L$-subset in RL-fuzzy topology based on the concepts of pseudocomplement of $L$-subsets and the implication operator. Since $L$-fuzzy topology in the sense of Kubiak and Šostak is a special case of RL-fuzzy topology, the degrees of RL-fuzzy compactness and the RL-Lindelöf property are generalization of the corresponding degrees in $L$-fuzzy topology.

\section{Preliminaries}

Throughout this study, $(L, \vee, \wedge, I)$ refers to a completely distributive lattice with order-reversing involution [2, 28], $X$ to a nonempty set, and $L^{X}$ to the collection of all $L$-subsets 
on $X$. The greatest and the smallest elements in $L$ and $L^{X}$ are 1,0 , and $1_{X}, 0_{X}$, respectively. For $\mu \in L^{X}, v \in L^{Y}$, and $f: X$ $\longrightarrow Y$, we have $f_{L} \longrightarrow(\mu)(y)=\vee\{\mu(x): f(x)=y\}$ for each $y \in Y$, and $f_{L}^{\leftarrow}(\nu)(x)=\vee\left\{\mu(x): f_{L} \longrightarrow(\mu) \leq \nu\right\}=\nu(f(x))$ for each $x \in X$. The binary operation $\mapsto$ on $L$ defined by $a \mapsto b=\vee\{c \in L \mid a \wedge c \leq b\}$ is called the implication operator. For more properties of the implication operator, we refer the reader to [29]. An $L$-subset $\lambda \in L^{X}$ is said to be valuable if $\lambda \leq \lambda^{\prime}$. The family of all valuable $L$-subsets on $X$ is denoted by $\mathscr{V}_{X}^{L}$, i.e., $\mathscr{V}_{X}^{L}=\left\{\lambda \in L^{X}: \lambda \leq \lambda \prime\right\}$. For all $\lambda \in \mathscr{V}_{X}^{L}$, we define the family $\mathscr{F}_{X}^{L}(\lambda)$ by $\mathscr{F}_{X}^{L}(\lambda)=\left\{\mu \in L^{X}: \mu \leq \lambda\right\}$. In fact, $\mathscr{F}_{X}^{L}(\lambda)$ represents the powerset of $L$-subset $\lambda \in L^{X}$. If $\lambda \in \mathscr{V}_{X}^{L}$ and $\mu \in \mathscr{V}_{Y}^{L}$, then the restriction of $f_{L} \longrightarrow$ on $\lambda$, i.e., $\left.f_{L} \longrightarrow\right|_{\lambda}: \mathscr{F}_{X}^{L}(\lambda) \longrightarrow L^{Y}$ such that $\gamma \in \mathscr{F}_{X}^{L}(A) \mapsto f_{L} \longrightarrow(\gamma)$, is called the relative $L$-fuzzy mapping (briefly, $R L$-fuzzy mapping) from $\lambda$ to $\mu$, denoted by $f_{L, \lambda}: \lambda \longrightarrow \mu$ if $f_{L} \longrightarrow(\lambda) \leq \mu$. The inverse image of an $L$-subset $\nu \in \mathscr{F}_{Y}^{L}(\mu)$ under $f_{L, \lambda}^{\longrightarrow}$ is given by $f_{L, \lambda}^{\leftarrow}(\nu)=\vee\left\{\gamma \in \mathscr{F}_{X}^{L}(\lambda)\right.$ : $f_{L} \longrightarrow(\gamma) \leq \nu$. Clearly, we have $f_{L, \lambda}^{\leftarrow}(\nu)=\lambda \wedge f_{L}^{\leftarrow}(\nu)$.

If $\lambda \in \mathscr{V}_{X}^{L}$ and $\mu \in \mathscr{F}_{X}^{L}(\lambda)$, the pseudocomplement of $\mu$ relative to $\lambda[26,27]$, denoted by $\left\langle{ }_{L}^{\lambda} \mu\right.$, is defined by

$$
\left\langle{ }_{L}^{\lambda} \mu= \begin{cases}\lambda \wedge \mu 1, & \text { if } \mu \neq \lambda, \\ 0_{X}, & \text { if } \mu=\lambda .\end{cases}\right.
$$

The following proposition lists some properties of pseudocomplement operation $\left\langle{ }_{L}^{\lambda}\right.$.

Proposition 1 (see [26, 27]). For each $\lambda \in \mathscr{V}_{X}^{L}$, $\mu, v \in \mathscr{F}_{X}^{L}(\lambda)$, and $\left\{\mu_{i}: i \in I\right\} \subseteq \mathscr{F}_{X}^{L}(\lambda)$, we have

(1) $\left\langle{ }_{L}^{\lambda} \mu=\lambda \Leftrightarrow \mu \leq \lambda\right.$.

(2) $\mu \leq \nu \Rightarrow\left\langle{ }_{L}^{\lambda} \nu \leq<{ }_{L}^{\lambda} \mu\right.$.

(3) $\left\langle{ }_{L}^{\lambda} \wedge_{i \in I} \mu_{i}=\vee_{i \in I}\left\langle{ }_{L}^{\lambda} \mu_{i}\right.\right.$.

(4) $\left\langle{ }_{L}^{\lambda} \vee_{i \in I} \mu_{i} \leq \Lambda_{i \in I}\left\langle{ }_{L}^{\lambda} \mu_{i}\right.\right.$. The equation holds provided that $\vee_{i \in I} \mu_{i} \neq \lambda$.

Definition 1. Let $\lambda \in \mathscr{V}_{X}^{L}$. A function ₹: $\mathscr{F}_{X}^{L}(\lambda) \times \mathscr{F}_{X}^{L}(\lambda)$ $\longrightarrow L$ is an RL-fuzzy inclusion on $X$, defined as $₹(\mu, \gamma)$ $=\wedge_{x \in X}\left(\left\langle\left\langle_{L}^{\lambda} \mu(x) \vee \gamma(x)\right)\right.\right.$, which is denoted by $[\mu \vDash \gamma]$ for simplicity instead of $₹(\mu, \gamma)$, i.e., $[\mu \vDash \gamma]=\wedge_{x \in X}\left(\left\langle_{L}^{\lambda} \mu(x) \vee\right.\right.$ $\gamma(x)$ ). Obviously, if $\lambda=1_{X}$, then $₹$ is an $L$-fuzzy inclusion function in the sense of Sostak [17].

The following lemma gives an important property for the RL-fuzzy mapping $f_{L, \lambda}$.

Lemma 1 (see [26]). Let $\lambda \in \mathscr{V}_{X}^{L}, \mu \in \mathscr{V}_{Y}^{L}$, and $f_{L, \lambda}: \lambda$ $\longrightarrow \mu$ be RL-fuzzy mapping from $\lambda$ to $\mu$, and $\gamma \in \mathscr{F}_{X}^{L}(\lambda)$. Then, for each $\mathscr{P} \subseteq L^{X}$, we have

$\bigvee_{y \in Y}\left(f_{L, \lambda}(\gamma)(y) \wedge \bigwedge_{\xi \in \mathscr{P}} \xi(y)\right)=\bigvee_{x \in X}\left(\gamma(x) \wedge \bigwedge_{\xi \in \mathscr{P}} f_{L, \lambda}^{\leftarrow}(\xi)(x)\right)$

Based on the properties of $\left\langle_{L}^{\lambda}\right.$ listed in Proposition 1, the previous equation can be rewritten as follows: $\bigwedge_{y \in Y}\left(\left\langle{ }_{L}^{\lambda} f_{L, \lambda}(\gamma)(y) \vee \bigvee_{\xi \in \mathscr{P}} \xi(y)\right)=\bigwedge_{x \in X}\left(\left\langle{ }_{L}^{\lambda} \gamma(x) \vee \bigvee_{\xi \in \mathscr{P}} f_{L, \lambda}^{\leftarrow}(\xi)(x)\right)\right.\right.$

An $L$-topological space $[1,2,28]$ (briefly, $L$-ts) is a pair $(X, \tau)$ such that $\tau$ is a subcollection of $L^{X}$ which contains $0_{X}$ and $1_{X}$ and is closed for any suprema and finite infima. Moreover, $\tau$ is called an $L$-topology on $X$. Furthermore, elements of $\tau$ are called open $L$-subsets, and their complements are called closed $L$-subsets. A mapping $f:(X, \tau) \longrightarrow(Y, \delta)$ is said to be $L$-continuous if and only if $f_{L}^{\leftarrow}(\nu) \in \tau$ for each $\nu \in \delta$.

Definition 2 (see $[10,11,30]$ ). An $L$-fuzzy topology on the set $X$ is the mapping $\tau: L^{X} \longrightarrow L$, which meets the following three conditions:

$$
\begin{aligned}
& \text { (T1) } \tau\left(0_{X}\right)=\tau\left(1_{X}\right)=1 . \\
& \text { (T2) } \forall \lambda, \mu \in L^{X}, \tau(\lambda \wedge \mu) \geq \tau(\lambda) \wedge \tau(\mu) . \\
& \text { (T3) } \forall\left\{\lambda_{i}: i \in I\right\} \subseteq L^{X}, \tau\left(\vee_{i \in I} \lambda_{i}\right) \geq \wedge_{i \in I} \tau\left(\lambda_{i}\right) .
\end{aligned}
$$

The pair $(X, \tau)$ is called an $L$-fuzzy topological space (briefly, $L$-fts). Here, $\tau(\lambda)$ can be regarded as the degree to which $\lambda$ is an open $L$-subset or the degree of openness of $\lambda$. Similarly, $\tau^{*}(\lambda)=\tau(\lambda \prime)$ can be regarded as the closeness degree of an $L$-subset $\lambda$. A mapping $f:(X, \tau) \longrightarrow(Y, \delta)$ between two $L$-fts's $(X, \tau)$ and $(Y, \delta)$ is said to be $L$-fuzzy continuous if and only if $\tau\left(f_{L}^{\leftarrow}(\nu)\right) \geq \delta(\nu)$ for each $\nu \in L^{Y}$.

The concept of RL-topology $x$ on an $L$-subset $\lambda$ is introduced as follows:

Definition 3 (see [26]). Let $\lambda \in \mathscr{V}_{X}^{L}$. A relative $L$-topology $x$ on an $L$-subset $\lambda$ is a subcollection on $\mathscr{F}_{X}^{L}(\lambda)$ that satisfies the following conditions:

(1) $\lambda \in \mathcal{\varkappa}$ and $\mu \in \mathcal{\varkappa}$ for all $\mu \leq \lambda$ '.

(2) $\mu_{1} \wedge \mu_{2} \in \varkappa$ for each $\mu_{1}, \mu_{2} \in \varkappa$.

(3) $\vee_{i \in I} \mu_{i} \in \mathcal{x}$ for each $\left\{\mu_{i}: i \in I\right\} \subseteq \mathcal{X}$.

The pair $(\lambda, \varkappa)$ is called a relative $L$-topological space on $\lambda$ (briefly, RL-ts). The members of $x$ are called relative open $L$-subsets (briefly, RL-open subset), and an $L$-subset $\mu$ is called relative $L$-closed (briefly, RL-closed subset) if and only if $\left\langle{ }_{L}^{\lambda} \mu \in \varkappa\right.$. The family of all RL-closed subsets with respect to $\varkappa$ is denoted by ${ }_{L}^{\lambda} \mathcal{x}$, i.e., $\left\langle{ }_{L}^{\lambda} \mathcal{\varkappa}=\left\{v:\left\langle{ }_{L}^{\lambda} v \in \mathcal{x}\right\}\right.\right.$. Let $\lambda \in \mathscr{V}_{X}^{L}$, $\mu \in \mathscr{V}_{Y}^{L}$, and $\left(\lambda, \varkappa_{1}\right),\left(\mu, \varkappa_{2}\right)$ be two RL-ts's. The relative fuzzy mapping $f_{L \lambda}: \lambda \longrightarrow \mu$ is said to be RL-continuous if and only if $f_{L, \lambda}^{\leftarrow}(\nu) \in\left\langle{ }_{L}^{\lambda} \varkappa_{1}\right.$ for each $v \in\left\langle{ }_{L}^{\lambda} \varkappa_{2}\right.$. It is not difficult to verify that the RL-topology on $\lambda$ degenerates to $L$-topology if $\lambda=1_{X}$.

Theorem 1 (see [26]). For any RL-ts $(\lambda, \varkappa)$, the following statements are true for $\left\langle_{L}^{\lambda} \varkappa\right.$ :

(1) $\lambda \in\left\langle{ }_{L}^{\lambda} \varkappa\right.$ and $\mu \in{ }_{L}^{\lambda} \varkappa$ for all $\mu \leq \lambda$.

(2) $\mu_{1} \vee \mu_{2} \in\left\langle{ }_{L}^{\lambda} \varkappa\right.$ for each $\mu_{1}, \mu_{2} \in\left\langle_{L}^{\lambda} \varkappa\right.$.

(3) $\wedge_{i \in I} \mu_{i} \in\left\langle L_{L}^{\lambda} \varkappa\right.$ for each $\left\{\mu_{i}: i \in I\right\} \subseteq\left\langle{ }_{L}^{\lambda} \varkappa\right.$. 
Definition 4 (see [27]). Let $\lambda \in \mathscr{V}_{X}^{L}$. An RL-fuzzy topology on $\lambda$ is a mapping $\varkappa$ : $\mathscr{F}_{X}^{L}(\lambda) \longrightarrow L$ such that $\varkappa$ satisfying the following statements:

$$
\begin{aligned}
& \text { (R1) } \varkappa(\lambda)=1, \forall \mu \leq \lambda \prime, \varkappa(\mu)=1 . \\
& \text { (R2) } \forall \mu_{1}, \mu_{2} \in \mathscr{F}_{X}^{L}(\lambda), \varkappa\left(\mu_{1} \wedge \mu_{2}\right) \geq \varkappa\left(\mu_{1}\right) \wedge \varkappa\left(\mu_{2}\right) . \\
& \text { (R3) } \forall\left\{\mu_{i}: i \in I\right\} \subseteq \mathscr{F}_{X}^{L}(\lambda), \varkappa\left(\vee_{i \in I} \mu_{i}\right) \geq \wedge_{i \in I} \varkappa\left(\mu_{i}\right) .
\end{aligned}
$$

The pair $(\lambda, \varkappa)$ is called an RL-fuzzy topological space (briefly, RL-fts) on $\lambda$. For each $\mu \in \mathscr{F}_{X}^{L}(\lambda)$, the values $\varkappa(\mu)$ (respectively, $x\left(\left\langle_{L}^{\lambda} \mu\right)\right.$ ) can be regarded as the degree of openness (respectively, closeness) of $\mu$ relative to $\varkappa$, respectively. Moreover, if $\varkappa(\mu)=1$ (respectively, $\varkappa\left(\left\langle_{L}^{\lambda} \mu\right)=1\right.$ ), then $\mu$ is called an RL-open (respectively, RL-closed) subset. It is easy to verify that when $\lambda=1_{X}$, the RL-fuzzy topology on $\lambda$ is reduced to $L$-fuzzy topology in the sense of Kubiak [10] and Šstak [11], that is, the RL-fuzzy topology on $\lambda$ is an extension of Kubiak-Šostak's $L$-fuzzy topology. Moreover, $(\lambda, \varkappa)$ is an RL-ts, and $\chi_{\varkappa}: \mathscr{F}_{X}^{L}(\lambda) \longrightarrow L$ is a mapping defined by

$$
\chi_{\varkappa}(\mu)= \begin{cases}1, & \text { if } \mu \in \mathcal{x}, \\ 0, & \text { if } \mu \notin \varkappa,\end{cases}
$$

Then, $\left(\lambda, \chi_{x}\right)$ can be seen as a special RL-fts. In this sense, $(\lambda, \varkappa)$ can also be regarded as RL-fts.

Theorem 2 (see [27]). For any $\lambda \in \mathscr{V}_{X}^{L}$ and $R L$-fts $(\lambda, \mathcal{x})$ on $\lambda$. The mapping $\left\langle{ }_{L}^{\lambda} \varkappa: \mathscr{F}_{X}^{L}(\lambda) \longrightarrow L\right.$ defined by $\left\langle{ }_{L}^{\lambda} \varkappa(\mu)=\right.$ $\varkappa\left(\left\langle_{L}^{\lambda} \mu\right)\right.$ for each $\mu \in \mathscr{F}_{X}^{L}(\lambda)$ satisfies the following statements:

$(R 1)^{\prime}\left\langle{ }_{L}^{\lambda} \varkappa(\lambda)=1, \forall \mu \leq \lambda\right.$, $\left\langle{ }_{L}^{\lambda} \varkappa(\mu)=1 ;\right.$

$(R 2)^{\prime} \quad \forall \mu_{1}, \quad \mu_{2} \in \mathscr{F}_{X}^{L}(\lambda), \quad\left\langle{ }_{L}^{\lambda} \varkappa\left(\mu_{1} \vee \mu_{2}\right) \geq\left\langle{ }_{L}^{\lambda} \varkappa\left(\mu_{1}\right) \wedge\right.\right.$ $\left\langle{ }_{L}^{\lambda} \mathcal{X}\left(\mu_{2}\right)\right.$;

$(R 3)^{\prime} \forall\left\{\mu_{i}: i \in I\right\} \subseteq \mathscr{F}_{X}^{L}(\lambda),\left\langle{ }_{L}^{\lambda} \varkappa\left(\wedge_{i \in I} \mu_{i}\right) \geq \wedge_{i \in I}\left\langle{ }_{L}^{\lambda} \varkappa\left(\mu_{i}\right)\right.\right.$.

$\pm b\left\langle_{L}^{\lambda} \kappa\right.$ is called an RL-fuzzy cotopology (briefly, RL-cft) on $\lambda$, and the pair $\left(\lambda,\left\langle{ }_{L}^{\lambda} \mathcal{x}\right)\right.$ is called an RL-fuzzy cotopological space (briefly, RL-cfts).

Definition 5 (see [27]). Let $\lambda \in \mathscr{V}_{X}^{L}, \mu \in \mathscr{V}_{Y}^{L}$, and $\left(\lambda, \varkappa_{1}\right)$, $\left(\mu, \varkappa_{2}\right)$ are RL-fts's on $\lambda$ and $\mu$, respectively. An RL-fuzzy mapping $f_{L, \lambda}: \lambda \longrightarrow \mu$ is said to be RL-fuzzy continuous if and only if $\varkappa_{1}\left(\left\langle_{L}^{\lambda} f_{L, \lambda}^{\leftarrow}(\nu)\right) \geq \varkappa_{1}\left(\left\langle_{L}^{\mu} \nu\right), \forall \nu \in \mathscr{F}_{Y}^{L}(\mu)\right.\right.$. Furthermore, if $\left(\lambda,\left\langle_{L}^{\lambda} \mathcal{\varkappa}_{1}\right)\right.$ and $\left(\mu,\left\langle{ }_{L}^{\mu} \varkappa_{2}\right)\right.$ are corresponding RL-cfts's of $\left(\lambda, \varkappa_{1}\right)$ and $\left(\mu, \varkappa_{2}\right)$, respectively; then, $f_{L, \lambda}$ is called an RL-fuzzy continuous if and only if $\left\langle_{L}^{\lambda} \varkappa_{1}\left(f_{L, \lambda}^{\leftarrow}(\nu)\right) \geq\left\langle{ }_{L}^{\mu} \varkappa_{2}(\nu)\right.\right.$, $\forall v \in \mathscr{F}_{X}^{L}(\mu)$.

Definition 6 (see [27]). Let $\lambda \in \mathscr{V}_{X}^{L}$ and $(\lambda, \varkappa)$ be an RL-fts on $\lambda$. An $L$-subset $\mu \in \mathscr{F}_{X}^{L}(\lambda)$ is said to be an RL-fuzzy compact with respect to $x$ if for each $\mathscr{P} \subseteq \mathscr{F}_{X}^{L}(\lambda)$, the following inequality holds:

$$
\begin{aligned}
& \bigvee_{\gamma \in \mathscr{P}} x\left(\left\langle{ }_{L}^{\lambda} \gamma\right) \vee \bigvee_{x \in X}\left(\mu(x) \wedge \bigwedge_{\gamma \in \mathscr{P}} \gamma(x)\right)\right. \\
& \geq \bigwedge_{\mathscr{R} \in 2^{\mathscr{P}}} \bigvee_{x \in X}\left(\mu(x) \wedge \bigwedge_{\gamma \in \mathscr{R}} \gamma(x)\right) .
\end{aligned}
$$

Theorem 3 (see [27]). In the case of $\lambda=1_{X}$, the following conclusions hold:

(1) $\left\langle{ }_{L}^{\lambda} \mu=\mu \prime, \mu \in \mathscr{F}_{X}^{L}(\lambda) \Leftrightarrow \mu \in L^{X}\right.$.

(2) $R L$-fuzzy compactness degenerates into L-fuzzy compactness.

(3) $\mu$ is RL-fuzzy compact if and only if $\mu$ is $L$-fuzzy compact.

Theorem 4 (see [27]). For any $\lambda \in \mathscr{V}_{X}^{L}$ and an $R L-f t x$ on $\lambda$, we have following conclusions:

(1) If $\mu_{1}, \mu_{2} \in \mathscr{F}_{X}^{L}(\lambda)$ such that $\mu_{1}$ and $\mu_{2}$ are $R L$-fuzzy

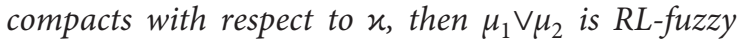
compact with respect to $x$.

(2) If $\mu_{1}, \mu_{2} \in \mathscr{F}_{X}^{L}(\lambda)$ such that $\mu_{1}$ is an RL-fuzzy compact with respect to $\varkappa$ and $\mu_{2}$ is an $R L$-closed subset, then $\mu_{1} \wedge \mu_{2}$ is an RL-fuzzy compact.

Definition 7. Let $\lambda \in \mathscr{V}_{X}^{L}, \quad x$ be an RL-ft on $\lambda$, and $\mu \in \mathscr{F}_{X}^{L}(\lambda)$. Then,

$$
\begin{aligned}
& \mathbf{c}_{\varkappa}(\mu)=\bigwedge_{\mathcal{U} \subseteq \mathscr{F}_{X}^{L}(\lambda)}\left\{\varkappa(\mathcal{U}) \mapsto\left\{[\mu \vDash \vee \mathscr{U}] \mapsto \bigvee_{\mathscr{V} \in 2^{(\mathcal{U})}}[\mu \vDash \vee \mathscr{V}]\right\}\right\} \\
& =\bigwedge_{\mathscr{U} \subseteq \mathscr{F}_{X}^{L}(\lambda)}\left\{\bigwedge _ { \gamma \in \mathscr { U } } x ( \gamma ) \mapsto \left\{\bigwedge _ { x \in X } \left(\left\langle{ }_{L}^{\lambda} \mu(x) \vee \bigvee_{\gamma \in \mathscr{U}} \gamma(x)\right)\right.\right.\right.
\end{aligned}
$$

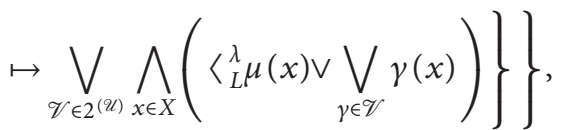

is called the RL-fuzzy compactness degree of $\mu$ with respect to $\varkappa$. Obviously, an $L$-subset $\mu$ is RL-fuzzy compact in RL-ts $\varkappa$ if and only if $\mathbf{c}_{\chi_{\varkappa}}(\mu)=1$.

Based on Definition 8 in [27], we can state the following theorem:

Theorem 5. For any $\lambda \in \mathscr{V}_{X}^{L} \lim _{x \longrightarrow \infty}, \mu \in \mathscr{F}_{X}^{L}(\lambda)$, and any mapping $x: \mathscr{F}_{X}^{L}(\lambda) \longrightarrow L$, let

$$
\begin{aligned}
\mathbf{c}_{\varkappa}(\mu)= & \bigwedge_{\mathscr{P} \subseteq \mathscr{F}_{X}^{L}(\lambda)}\left\{\left\{\bigwedge_{\mathscr{F} \in 2^{(\mathscr{P})}} \bigvee_{x \in X}\left(\mu(x) \wedge \bigwedge_{\gamma \in \mathscr{F}} \gamma(x)\right)\right.\right. \\
& \left.\mapsto \bigvee_{x \in X}\left(\mu(x) \wedge \bigwedge_{\gamma \in \mathscr{P}} \gamma(x)\right)\right\} \mapsto\left\langle{ }_{L}^{\lambda} x(\mathscr{P})\right\} \\
= & \bigwedge_{\mathscr{P} \subseteq \mathscr{F}_{X}^{L}(\lambda)}\left\{\left\{\bigwedge_{\mathscr{F} \in 2^{(\mathscr{P})}} \bigvee_{x \in X}\left(\mu(x) \wedge \bigwedge_{\gamma \in \mathscr{F}} \gamma(x)\right)\right.\right. \\
& \left.\left.\mapsto \bigvee_{x \in X}\left(\mu(x) \wedge \bigwedge_{\gamma \in \mathscr{P}} \gamma(x)\right)\right\} \mapsto \bigvee_{\gamma \in \mathscr{P}} x\left({ }_{L}^{\lambda} \gamma\right)\right\}
\end{aligned}
$$

If $(\lambda, \varkappa)$ be an RL-fts, then $\mathbf{c}_{\varkappa}(\mu)$ is called the RL-fuzzy compactness degree of $\mu$ with respect to $\varkappa$. Clearly, $\mu$ is the RL-fuzzy compact in RL-ts $\varkappa$ iff $\mathbf{c}_{\chi_{x}}(\mu)=1$. 


\section{Measure of RL-Countable Compactness}

Let $\tilde{\mathscr{F}}_{X}^{L}(\lambda)=\left\{\mathcal{U} \subseteq \mathscr{F}_{X}^{L}(\lambda) \mid \mathcal{U}\right.$ is a countable $\}$. If $\lambda \in \mathscr{V}_{X}^{L}$, $(\lambda, \mathcal{x})$ be an RL-ts on $\lambda$, and $\varkappa_{c}=\{\mathscr{U} \subseteq \mathcal{x} \mid \mathscr{U}$ is a countable family\}. Then, $\mu \in \mathscr{F}_{X}^{L}(\lambda)$ is countably RL-compact if and only if for each $\mathscr{U} \subseteq \mathcal{K}_{c}$, it follows that $[\mu \vDash \vee \mathscr{U}] \leq \vee_{\left.\mathscr{V} \in 2^{(\mathscr{}}\right)}[\mu \gtrless \vee \mathscr{V}]$. This implies that $[[\mu \vDash \vee \mathscr{U}] \leq$
$\left.\vee_{\mathscr{V} \in 2^{(\ell)}}[\mu \gtrless \vee \mathscr{V}]\right]=1$, i.e., $\quad \mu \in \mathscr{F}_{X}^{L}(\lambda) \quad$ is countably RL-compact if and only if for each collection $\mathscr{U} \subseteq \mathcal{\varkappa}_{c}$, it follows that $\chi_{\varkappa}(\mathscr{U}) \leq\left[[\mu \gtrless \vee \mathscr{U}] \leq \mathrm{V}_{\mathscr{V} \in 2^{(\mathcal{U})}}[\mu \gtrless \vee \mathscr{V}]\right]$.

Definition 8. Let $\lambda \in \mathscr{V}_{X}^{L}, \quad \varkappa$ be an RL-ft on $\lambda$, and $\mu \in \mathscr{F}_{X}^{L}(\lambda)$. Then,

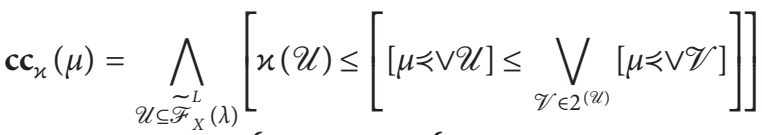

$$
\begin{aligned}
& =\bigwedge_{\mathcal{U} \subseteq \widetilde{\mathscr{F}}_{X}^{L}(\lambda)}\left\{\bigwedge _ { \gamma \in \mathscr { U } } x ( \gamma ) \mapsto \left\{\bigwedge_{x \in X}\left(\left\langle{ }_{L}^{\lambda} \mu(x) \vee \bigvee_{\gamma \in \mathscr{U}} \gamma(x)\right) \mapsto \bigvee_{\mathscr{V} \in 2^{(\mathscr{U})}} \bigwedge_{x \in X}\left(\left\langle{ }_{L}^{\lambda} \mu(x) \vee \bigvee_{\gamma \in \mathscr{V}} \gamma(x)\right)\right\}\right\}\right.\right. \text {, }
\end{aligned}
$$

is the degree of countable RL-compactness of $\mu$ with respect to $\varkappa$. Clearly, $\mu$ is the countable RL-compact in RL-ts $(\lambda, \varkappa)$ if and only if $\mathrm{cc}_{\chi_{\varkappa}}(\mu)=1$. Based on the properties of implication operation “ $\mapsto$ " ([29]), we can state the following lemma:

Lemma 2. Let $\lambda \in \mathscr{V}_{X}^{L},(\lambda, \varkappa)$ be an RL-fts on $\lambda$, and $\mu \in \mathscr{F}_{X}^{L}(\lambda)$. Then, $\alpha \leq \mathbf{c c}_{\varkappa}(\mu)$ if and only if

$$
\varkappa(\mathscr{U}) \wedge[\mu \gtrless \vee \mathscr{U}] \wedge \alpha \leq \bigvee_{\left.\mathscr{V} \in 2^{(\mathscr{}}\right)}[\mu \gtrless \vee \mathscr{V}]
$$

for each $\mathcal{U \subseteq \widetilde { F } _ { X } ^ { L }}(\lambda)$

Theorem 6. Let $\lambda \in \mathscr{V}_{X}^{L},(\lambda, \varkappa)$ be an RL-fts on $\lambda$, and $\mu \in \mathscr{F}_{X}^{L}(\lambda)$. Then,

$$
\mathbf{c c}_{\varkappa}(\mu)=\vee\left\{\alpha \in L \mid \mathcal{x}(\mathscr{U}) \wedge[\mu \gtrless \vee \mathscr{U}] \wedge \alpha \leq \bigvee_{\mathscr{V} \in 2^{(\mathscr{U})}}[\mu \vDash \vee \mathscr{V}], \quad \forall \mathscr{U} \subseteq \widetilde{\mathscr{F}}_{X}^{L}(\lambda)\right\}
$$

The following theorem is an intuitive result from the Definitions 7 and 8.

Theorem 7. Let $\lambda \in \mathscr{V}_{X}^{L},(\lambda, \varkappa)$ be an $R L$-fts on $\lambda$, and $\mu \in \mathscr{F}_{X}^{L}(\lambda)$. Then, $\mathbf{c}_{\varkappa}(\mu) \leq \mathbf{c c}_{\varkappa}(\mu)$.

Theorem 8. Let $\lambda \in \mathscr{V}_{X}^{L},(\lambda, \varkappa)$ be an RL-fts on $\lambda$, and $\mu_{1}, \mu_{2} \in \mathscr{F}_{X}^{L}(\lambda)$. Then, $\operatorname{cc}_{\varkappa}\left(\mu_{1} \wedge \mu_{2}\right) \geq \mathbf{c c}_{\varkappa}\left(\mu_{1}\right) \wedge\left\langle{ }_{L}^{\lambda} \varkappa\left(\mu_{2}\right)\right.$.
Proof. Suppose that $\mathrm{cc}_{\varkappa}\left(\mu_{1}\right) \wedge\left\langle{ }_{L}^{\lambda} \varkappa\left(\mu_{2}\right) \geq \alpha\right.$ for any $\alpha \in L$ and $\mathcal{U} \subseteq \widetilde{\mathscr{F}}_{X}^{L}(\lambda)$, then $\left\langle{ }_{L}^{\lambda} \varkappa\left(\mu_{2}\right) \geq \alpha\right.$ and $\operatorname{cc}_{\varkappa}\left(\mu_{1}\right) \geq \alpha$. Consider $\mathscr{W}=\mathscr{U} \cup\left\langle{ }_{L}^{\lambda} \mu_{2}\right.$, then $\mathscr{W} \subseteq \widetilde{\mathscr{F}}_{X}^{L}(\lambda)$. By Lemma 2, $\varkappa(\mathscr{W})$ $\wedge\left[\mu_{1} \preccurlyeq \vee \mathscr{W}\right] \wedge \alpha \leq \vee_{\left.\mathscr{V} \in 2^{(\mathscr{W}}\right)}\left[\mu_{1} \preccurlyeq \vee \mathscr{V}\right]$. Since $\varkappa(\mathscr{W})=\varkappa(\mathscr{U}) \wedge$ $\varkappa\left(<{ }_{L}^{\lambda} \mu_{2}\right)=\varkappa(\mathcal{U}) \wedge\left\langle{ }_{L}^{\lambda} \varkappa\left(\mu_{2}\right)\right.$, we have

$$
\begin{aligned}
& {\left[\mu_{1} \vDash \vee \mathscr{W}\right]=\bigwedge_{x \in X}\left(\left\langle{ }_{L}^{\lambda} \mu_{1}(x) \vee \bigvee_{\gamma \in \mathscr{W}} \gamma(x)\right)=\bigwedge_{x \in X}\left(\left\langle{ }_{L}^{\lambda} \mu_{1}(x) \vee\left\langle{ }_{L}^{\lambda} \mu_{2}(x) \vee \bigvee_{\gamma \in \mathscr{U}} \gamma(x)\right)\right.\right.\right.} \\
& =\bigwedge_{x \in X}\left(\left\langle_{L}^{\lambda}\left(\mu_{1}(x) \wedge \mu_{2}(x)\right) \vee \bigvee_{\gamma \in \mathscr{U}} \gamma(x)\right)=\left[\left\langle_{L}^{\lambda}\left(\mu_{1} \wedge \mu_{2}\right) \gtrless \vee \mathcal{U}\right],\right.\right. \\
& \bigvee_{\mathscr{V} \in 2^{(\mathscr{W})}}\left[\mu_{1} \vDash \vee \mathscr{V}\right]=\bigvee_{\mathscr{V} \in 2^{(\mathscr{U})}}\left[\mu_{1} \vDash \vee \mathscr{V}\right] \vee \bigvee_{\mathscr{V} \in 2^{(\mathscr{U})}}\left[\mu_{1} \vDash \vee\left(\mathscr{V} \vee\left\langle{ }_{L}^{\lambda} \mu_{2}\right)\right]\right.
\end{aligned}
$$

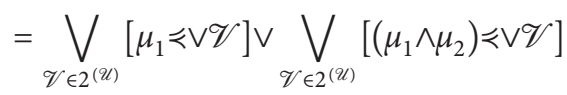

$$
\begin{aligned}
& =\bigvee_{\left.\mathscr{V} \in 2^{(\mathscr{}}\right)}\left[\left(\mu_{1} \wedge \mu_{2}\right) \gtrless \vee \mathscr{V}\right] \text {. }
\end{aligned}
$$


Therefore, $\quad\left(\varkappa(\mathscr{U}) \wedge\left\langle{ }_{L}^{\lambda} \varkappa\left(\mu_{2}\right)\right) \wedge\left[\left(\mu_{1} \wedge \mu_{2}\right) ₹ \vee \mathscr{V}\right] \wedge \alpha \leq\right.$ $\vee_{\mathscr{V} \in 2^{(\ell)}}\left[\left(\mu_{1} \wedge \mu_{2}\right) \gtrless \vee \mathscr{V}\right]$. Since $\left\langle{ }_{L}^{\lambda} \mathcal{\varkappa}\left(\mu_{2}\right) \geq \alpha\right.$, we have $\varkappa(\mathscr{U}) \wedge\left[\left(\mu_{1} \wedge \mu_{2}\right) \gtrless \vee \mathscr{U}\right] \wedge \alpha \leq \vee_{\mathscr{V} \in 2^{(}(\mathcal{U})}\left[\left(\mu_{1} \wedge \mu_{2}\right) \gtrless \vee \mathscr{V}\right]$. Thus, by Lemma 2, $\quad \operatorname{cc}_{\varkappa}\left(\mu_{1} \wedge \mu_{2}\right) \geq \alpha$, and the proof is completed.

Corollary 1. Let $\lambda \in \mathscr{V}_{X}^{L},(\lambda, \varkappa)$ be an RL-fts on $\lambda$, and $\mu \in \mathscr{F}_{X}^{L}(\lambda)$. Then, $\operatorname{cc}_{\varkappa}(\mu) \geq \mathbf{c c}\left(1_{X}\right) \wedge\left\langle{ }_{L}^{\lambda}(\mu)\right.$.
Theorem 9. Let $\lambda \in \mathscr{V}_{X}^{L},(\lambda, \varkappa)$ be an RL-fts on $\lambda$, and $\mu_{1}, \mu_{2} \in \mathscr{F}_{X}^{L}(\lambda)$. Then, $\operatorname{cc}_{\varkappa}\left(\mu_{1} \vee \mu_{2}\right) \geq \mathbf{c c}_{\varkappa}\left(\mu_{1}\right) \wedge \mathbf{c c}_{\varkappa}\left(\mu_{2}\right)$.

Proof. Suppose that $\operatorname{cc}_{\varkappa}\left(\mu_{1}\right) \wedge \mathbf{c c}_{\varkappa}\left(\mu_{2}\right) \geq \alpha$ for each $\alpha \in L$ and $U \subseteq \widetilde{\mathscr{F}}_{X}^{L}(\lambda)$, then $\operatorname{cc}_{\varkappa}\left(\mu_{1}\right) \geq \alpha$ and $\operatorname{cc}_{\varkappa}\left(\mu_{2}\right) \geq \alpha$. By using Lemma 2 , we have $x(\mathscr{U}) \wedge\left[\mu_{1} \vDash \vee \mathscr{U}\right] \wedge \alpha \leq \vee_{\mathscr{V} \in 2^{(}(x)}\left[\mu_{1} \vDash \vee \mathscr{V}\right]$ and $\quad \varkappa(\mathscr{U}) \wedge\left[\mu_{2} \vDash \vee \mathscr{U}\right] \wedge \alpha \leq \mathrm{V}_{\mathscr{V} \in 2(\mathcal{Q})}\left[\mu_{2} \vDash \vee \mathscr{V}\right]$. Therefore, $\varkappa(\mathscr{U}) \wedge \quad\left[\mu_{1} \vDash \vee \mathscr{U}\right] \wedge\left[\mu_{2} ₹ \vee \mathscr{U}\right] \wedge \alpha \leq\left(\vee_{\mathscr{V} \in 2^{(2)}}\left[\mu_{1} ₹ \vee \mathscr{V}\right]\right) \wedge$ $\left(\vee_{\mathscr{V} \in 2^{(\varkappa)}}\left[\mu_{2} \preccurlyeq \vee \mathscr{V}\right]\right)$. By

$$
\begin{aligned}
& {\left[\mu_{1} \vDash \vee \mathcal{U}\right] \wedge\left[\mu_{2} ₹ \vee \mathcal{U}\right]=\left(\bigwedge _ { x \in X } ( \langle { } _ { L } ^ { \lambda } \mu _ { 1 } ( x ) \vee \bigvee _ { \gamma \in \mathcal { U } } \gamma ( x ) ) ) \wedge \left(\bigwedge_{x \in X}\left(\left\langle{ }_{L}^{\lambda} \mu_{2}(x) \vee \bigvee_{\gamma \in \mathscr{U}} \gamma(x)\right)\right)\right.\right.} \\
& =\bigwedge_{x \in X}\left(( \langle { } _ { L } ^ { \lambda } \mu _ { 1 } ( x ) \vee \bigvee _ { \gamma \in \mathscr { U } } \gamma ( x ) ) ) \wedge \left(\left(\left\langle{ }_{L}^{\lambda} \mu_{2}(x) \vee \bigvee_{\gamma \in \mathscr{U}} \gamma(x)\right)\right)\right.\right. \\
& =\left(\bigwedge_{x \in X}\left(\left(\left\langle{ }_{L}^{\lambda} \mu_{1}(x) \wedge<{ }_{L}^{\lambda} \mu_{2}(x)\right) \vee \bigvee_{\gamma \in \mathcal{U}} \gamma(x)\right)\right)\right. \\
& =\left(\bigwedge_{x \in X}\left(\left\langle{ }_{L}^{\lambda}\left(\mu_{1} \vee \mu_{2}\right)(x) \vee \bigvee_{\gamma \in \mathscr{U}} \gamma(x)\right)\right)\right. \\
& =\left[\left(\mu_{1} \vee \mu_{2}\right) \gtrless \vee \mathcal{U}\right] \text {, }
\end{aligned}
$$

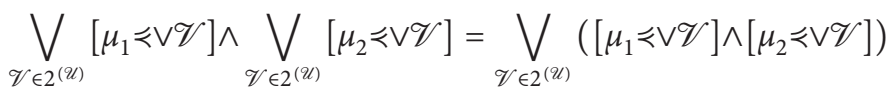

$$
\begin{aligned}
& =\bigvee_{\mathscr{V} \in 2^{(\mathscr{U})}}\left[\left(\mu_{1} \vee \mu_{2}\right) \gtrless \vee \mathscr{V}\right]
\end{aligned}
$$

we have that $\quad \varkappa(\mathscr{U}) \wedge\left[\left(\mu_{1} \vee \mu_{2}\right) \gtrless \vee \mathscr{U}\right] \wedge \alpha \leq \vee_{\left.\mathscr{V} \in 2^{(}()\right)}$ $\left[\left(\mu_{1} \vee \mu_{2}\right) \gtrless \vee \mathscr{V}\right]$. By using Lemma 2, we can obtain that cc $_{\varkappa}\left(\mu_{1} \vee \mu_{2}\right) \geq \alpha$, and thus, the proof is completed.

Theorem 10. Let $\lambda \in \mathscr{V}_{X}^{L}, \mu \in \mathscr{V}_{Y}^{L},\left(\lambda, \varkappa_{1}\right)$, and $\left(\mu, \varkappa_{2}\right)$ be $R L$-fts. If $f_{L \lambda}: \lambda \longrightarrow \mu$ is RL-fuzzy continuous, then $\operatorname{cc}_{\varkappa_{2}}(f \overrightarrow{L, \lambda}(\nu)) \geq \mathbf{c c}_{\varkappa_{1}}(\nu)$.

Proof. Suppose that $\operatorname{cc}_{\varkappa_{1}}(\nu) \geq \alpha$ for each $\alpha \in L$, and let

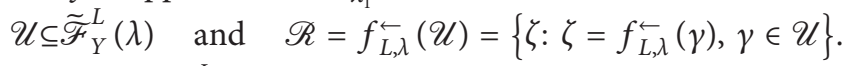
Then, $\mathscr{R} \subseteq \widetilde{\mathscr{F}}_{X}^{L}(\lambda)$. Since $\mathrm{cc}_{\varkappa_{1}}(\nu) \geq \alpha$, we have $\varkappa_{1}(\mathscr{R}) \wedge$ $[\nu \gtrless \vee \mathscr{R}] \wedge \alpha \leq \vee_{\mathcal{S} \in 2^{(\mathscr{R})}}[\nu \prec \vee \mathcal{S}]$. Since $f_{L, \lambda}$ is RL-fuzzy continuous, $\quad \varkappa_{1}\left(f_{L, \lambda}^{\leftarrow}(\gamma)\right) \geq \mathcal{x}_{2}(\gamma)$ for each $\gamma \in \mathcal{U}$, i.e., $\varkappa_{1}(\mathscr{R})=\varkappa_{1}\left(f_{L, \lambda}^{\leftarrow}(\mathscr{U})\right) \geq \varkappa_{2}(\mathscr{U})$. Based on Lemma 1, we have

$$
\begin{aligned}
& {\left[f_{L, \lambda}(\nu) ₹ \vee \mathcal{U}\right]=\bigwedge_{y \in Y}\left(\left\langle{ }_{L}^{\lambda} f_{L, \lambda}(\nu)(y) \vee \bigvee_{\gamma \in \mathscr{U}} \gamma(y)\right)\right.} \\
& =\bigwedge_{x \in X}\left(\left\langle{ }_{L}^{\lambda} \nu(x) \vee \bigvee_{\eta \in \mathscr{R}} \eta(x)\right)\right. \\
& =[\nu \gtrless \vee \mathscr{R}] \text {, } \\
& \bigvee_{\mathcal{S} \in 2^{(\mathscr{S})}}[\nu \vDash \vee \mathcal{S}]=\bigvee_{\delta \in 2^{(\mathscr{S})}} \bigwedge_{x \in X}\left(\left\langle_{L}^{\lambda} \nu(x) \vee \bigvee_{\xi \in \mathcal{S}} \xi(x)\right)\right. \\
& =\bigvee_{\left.\mathscr{V} \in 2^{(2)}\right)} \bigwedge_{y \in Y}\left(\left\langle_{L}^{\lambda} f_{L, \lambda} \vec{l}(\nu)(y) \vee \bigvee_{\psi \in \mathscr{V}} \psi(y)\right)\right. \\
& =\bigvee_{\left.\mathscr{V} \in 2^{(\mathcal{}}\right)}\left[f_{L, \lambda}(\nu) \gtrless \vee \mathscr{V}\right] \text {. }
\end{aligned}
$$


Therefore, we have the following inequality:

$$
\begin{aligned}
\varkappa_{2}(\mathcal{U}) \wedge\left[f_{L, \lambda}(\nu) \preccurlyeq \vee \mathcal{U}\right] \wedge \alpha & \leq \varkappa_{1}(\mathscr{R}) \wedge[\nu \vDash \vee \mathscr{R}] \wedge \alpha \\
& \leq \bigvee_{\delta \in 2^{(\mathscr{R})}}[\nu \vDash \vee \mathcal{S}] \\
& =\bigvee_{\mathscr{V} \in 2^{(\mathscr{U})}}\left[f_{L, \lambda} \vec{\longrightarrow}(\nu) \gtrless \vee \mathscr{V}\right] .
\end{aligned}
$$

Thus, by Lemma $2, \mathbf{c c}_{\mathcal{\varkappa}_{2}}\left(f_{L, \lambda}(\nu)\right) \geq \alpha$, and thus completed the proof.

\section{Lindelöf Property Degree in RL-Fts's}

Definition 9. Let $\lambda \in \mathscr{V}_{X}^{L},(\lambda, \varkappa)$ be an RL-fts on $\lambda$, and $\mu \in \mathscr{F}_{X}^{L}(\lambda)$. Then,

$$
\begin{aligned}
& \mathscr{L}_{\varkappa}(\mu)=\bigwedge_{\mathscr{U} \subseteq \mathscr{F}_{X}^{L}(\lambda)}\left[\varkappa(\mathscr{U}) \leq\left[[\mu \gtrless \vee \mathscr{U}] \leq \bigvee_{\mathscr{V} \in 2^{[\mathscr{U}]}}[\mu \gtrless \mathrm{V} \mathscr{V}]\right]\right] \\
& =\bigwedge_{\mathscr{U} \subseteq \mathscr{F}_{X}^{L}(\lambda)}\left\{\bigwedge _ { \gamma \in \mathscr { U } } x ( \gamma ) \mapsto \left\{\bigwedge _ { x \in X } \left(\left\langle{ }_{L}^{\lambda} \mu(x) \vee \bigvee_{\gamma \in \mathscr{U}} \gamma(x)\right)\right.\right.\right. \\
& \left.\mapsto \bigvee_{\mathscr{V} \in 2^{[\mathscr{U}]}} \bigwedge_{x \in X}\left(\left\langle{ }_{L}^{\lambda} \mu(x) \vee \bigvee_{\gamma \in \mathscr{V}} \gamma(x)\right)\right\}\right\},
\end{aligned}
$$

is the degree to which $\mu$ has the RL-Lindelöf property with respect to $\varkappa$. Clearly, $\mu$ has the RL-Lindelöf property in RL-ts $\varkappa$ if and only if $\mathscr{L}_{\chi_{\varkappa}}(\mu)=1$.

Lemma 3. Let $\lambda \in \mathscr{V}_{X}^{L},(\lambda, \varkappa)$ be an RL-fts on $\lambda$, and $\mu \in \mathscr{F}_{X}^{L}(\lambda)$. Then, $\alpha \leq \mathscr{L}_{\varkappa}(\mu)$ if and only if

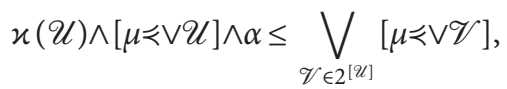

for each $\mathscr{U} \subseteq \mathscr{F}_{X}^{L}(\lambda)$.

Theorem 11. Let $\lambda \in \mathscr{V}_{X}^{L},(\lambda, \varkappa)$ be an RL-fts on $\lambda$, and $\mu \in \mathscr{F}_{X}^{L}(\lambda)$. Then,

$$
\mathscr{L}_{\varkappa}(\mu)=\vee\left\{\alpha \in L \mid \varkappa(\mathcal{U}) \wedge[\mu \vDash \vee \mathscr{U}] \wedge \alpha \leq \bigvee_{\mathscr{V} \in 2^{[\mathscr{U}]}}[\mu \gtrless \vee \mathscr{V}], \quad \forall \mathscr{U} \subseteq \mathscr{F}_{X}^{L}(\lambda)\right\}
$$

Theorem 12. Let $\lambda \in \mathscr{V}_{X}^{L},(\lambda, \varkappa)$ be an RL-fts on $\lambda$, and $\mu_{1}, \mu_{2} \in \mathscr{F}_{X}^{L}(\lambda)$. Then, $\mathscr{L}_{\varkappa}^{X}\left(\mu_{1} \wedge \mu_{2}\right) \geq \mathscr{L}_{\varkappa}\left(\mu_{1}\right) \wedge<{ }_{L}^{\lambda} \varkappa\left(\mu_{2}\right)$.

Corollary 2. Let $\lambda \in \mathscr{V}_{X}^{L},(\lambda, \varkappa)$ be an RL-fts on $\lambda$, and $\mu \in \mathscr{F}_{X}^{L}(\lambda)$. Then, $\mathscr{L}_{\varkappa}(\mu) \geq \mathscr{L}\left(1_{X}\right) \wedge\left\langle{ }_{L}^{\lambda}(\mu)\right.$.

Theorem 13. Let $\lambda \in \mathscr{V}_{X}^{L},(\lambda, \varkappa)$ be an RL-fts on $\lambda$, and $\mu_{1}, \mu_{2} \in \mathscr{F}_{X}^{L}(\lambda)$. Then, $\mathscr{L}_{\varkappa}^{X}\left(\mu_{1} \vee \mu_{2}\right) \geq \mathscr{L}_{\varkappa}\left(\mu_{1}\right) \wedge \mathscr{L}_{\varkappa}\left(\mu_{2}\right)$.

Theorem 14. Let $\lambda \in \mathscr{V}_{X}^{L},(\lambda, \varkappa)$ be an RL-fts on $\lambda$, and $\mu \in \mathscr{F}_{X}^{L}(\lambda)$. Then, $\mathscr{L}_{\varkappa}(\mu) \wedge \mathrm{cc}_{\varkappa}(\mu) \leq \mathbf{c}_{\varkappa}(\mu)$.

Proof. Suppose $\mathscr{L}_{\varkappa}(\mu) \wedge c_{\varkappa}(\mu) \geq \alpha$ for each $\alpha \in L$ and $\mathscr{U} \subseteq \mathscr{F}_{X}^{L}(\lambda)$. Then, $\mathscr{L}_{\varkappa}(\mu) \geq \alpha$ and $\mathbf{c c}_{\varkappa}(\mu) \geq \alpha$. Thus,

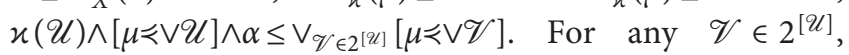
$\mathscr{V} \subseteq \widetilde{\mathscr{F}}_{X}^{L}(\lambda)$. Therefore, $\boldsymbol{\varkappa}(\mathcal{U}) \leq \varkappa(\mathscr{V})$, and by using Lemma 2 , we have $\varkappa(\mathscr{V}) \wedge[\mu \vDash \vee \mathscr{V}] \wedge \alpha \leq \vee_{\mathscr{W} \in 2(\mathscr{V})}[\mu \gtrless \vee \mathscr{W}]$. Moreover, we have

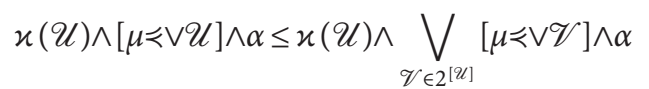

$$
\begin{aligned}
& \leq \bigvee_{\mathscr{V} \in 2^{[\mathscr{U}]}}(\varkappa(\mathscr{V}) \wedge[\mu \preccurlyeq \vee \mathscr{V}] \wedge \alpha) \\
& \leq \bigvee_{\mathscr{V} \in 2^{[\mathscr{U}]}}\left(\bigvee_{\mathscr{W} \in 2^{(\mathscr{V})}}[\mu \gtrless \vee \mathscr{W}]\right) \\
& \leq \bigvee_{\mathscr{V} \in 2^{[\mathscr{U}]}}\left(\bigvee_{\mathscr{W} \in 2^{(\mathscr{U})}}[\mu \gtrless \vee \mathscr{W}]\right) \\
& \leq \bigvee_{\mathscr{W} \in 2^{(\mathscr{V})}}[\mu \vDash \vee \mathscr{W}]=\bigvee_{\mathscr{V} \in 2^{(\mathscr{U})}}[\mu \gtrless \vee \mathscr{V}] .
\end{aligned}
$$

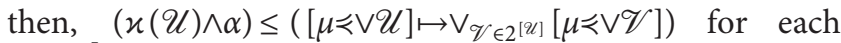

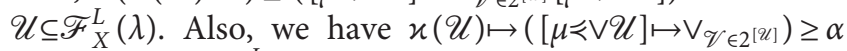
for each $\mathcal{U} \subseteq \mathscr{F}_{X}^{L}(\lambda)$. Hence, $\mathbf{c}_{\varkappa}(\mu) \geq \alpha$. The proof is completed.

The following corollary can be considered as the multivalue extension of the principal. If $\mu$ has the Lindelöf property, then $\mu$ is compact if and only if it is countable compact. 
Corollary 3. Let $\lambda \in \mathscr{V}_{X}^{L},(\lambda, \varkappa)$ be an $R L$-fts on $\lambda$, and $\mu \in \mathscr{F}_{X}^{L}(\lambda)$. Then, $\mathscr{L}_{\varkappa}(\mu) \wedge \mathbf{c c}_{\varkappa}(\mu) \leq \mathscr{L}_{\varkappa}(\mu) \wedge \mathbf{c}_{\varkappa}(\mu)$.

\section{Conclusion}

In this paper, we introduced and characterized the countable RL-fuzzy compactness degree and Lindelöf property degree of an $L$-subset in RL-fuzzy topology based on pseudocomplement of $L$-subsets and an implication operator. Since if $\lambda=1_{X}$, the $R L$-fuzzy topology on $\lambda$ is reduced to $L$-fuzzy topology in the sense of Kubiak and Šostak; the degrees of RL-fuzzy compactness and Lindelöf property are generalizations of the corresponding degrees in $L$-fuzzy topology.

\section{Data Availability}

No data were used to support this study.

\section{Conflicts of Interest}

The authors declare that they have no conflicts of interest.

\section{Acknowledgments}

The authors extend appreciation to the Deanship of Scientific Research, University of Hafr Al Batin, for funding this work (G-104-2020) and the Science and Technology Project of Yulin City (CXY-2020-007).

\section{References}

[1] C. L. Chang, "Fuzzy topological spaces," Journal of Mathematical Analysis and Applications, vol. 24, no. 1, pp. 182-190, 1968.

[2] L. Ying-Ming and L. Mao-Kang, Fuzzy Topology, World Scientific, Singapore, 1998.

[3] R. Lowen, "A comparison of different compactness notions in fuzzy topological spaces," Journal of Mathematical Analysis and Applications, vol. 64, no. 2, pp. 446-454, 1978.

[4] F.-G. Shi, "Countable compactness and the lindelöf property of L-fuzzy sets," Iranian Journal of Fuzzy Systems, vol. 1, no. 1, pp. 79-88, 2004.

[5] F.-G. Shi, "A new notion of fuzzy compactness in $L$-topological spaces," Information Sciences, vol. 173, no. 1-3, pp. 35-48, 2005.

[6] F.-G. Shi, "A new definition of fuzzy compactness," Fuzzy Sets and Systems, vol. 158, no. 13, pp. 1486-1495, 2007.

[7] A. Ghareeb, "A general framework for compactness in L-topological spaces," Hacettepe Journal of Mathematics and Statistics, vol. 41, pp. 67-75, 2012.

[8] A. M. Zahran, A. Ghareeb, and A. H. Zakari, " $\gamma$-compactness in L-topological spaces," Kragujevac Journal of Mathematics, vol. 35 , pp. 25-37, 2011.

[9] U. Höhle, "Upper semicontinuous fuzzy sets and applications," Journal of Mathematical Analysis and Applications, vol. 78, no. 2, pp. 659-673, 1980.

[10] T. Kubiak, On fuzzy topologies, Ph.D. thesis, Adam Mickiewicz, Poznan, Poland, 1985.

[11] A. P. Šostak, "On a fuzzy topological structure," Rendiconti Circolo Matematico Palermo, vol. 11, pp. 89-103, 1985.

[12] A. H. Eş and D. Çoker, "On several types of degree of fuzzy compactness," Fuzzy Sets and Systems, vol. 87, no. 3, pp. 349-359, 1997.
[13] F. Jinming, "Categories isomorphic to -FTOP," Fuzzy Sets and Systems, vol. 157, no. 6, pp. 820-831, 2006.

[14] G. Jäger, "Degrees of compactness in fuzzy convergence spaces," Fuzzy Sets and Systems, vol. 125, no. 2, pp. 167-175, 2002.

[15] H.-Y. Li and F.-G. Shi, "Measures of fuzzy compactness in L-fuzzy topological spaces," Computers \& Mathematics with Applications, vol. 59, no. 2, pp. 941-947, 2010.

[16] E. Lowen and R. Lowen, "On measures of compactness in fuzzy topological spaces," Journal of Mathematical Analysis and Applications, vol. 131, no. 2, pp. 329-340, 1988.

[17] A. Šstak, General Topology and its Relations to Modern Analysis and Algebra, Chapter on Compactness and Connectedness Degrees of Fuzzy Sets in Fuzzy Topological Spaces, Heldermann Verlag, Berlin, Germany, 1988.

[18] Y. Yueli and F. Jinming, "Generated I-fuzzy topological spaces," Fuzzy Sets and Systems, vol. 154, no. 1, pp. 103-117, 2005.

[19] A. Ghareeb, " $L$-fuzzy semi-preopen operator in $L$-fuzzy topological spaces," Neural Computing and Applications, vol. 21, no. 1, pp. 87-92, 2012.

[20] A. Ghareeb, "A new form of $F$-compactness in $L$-fuzzy topological spaces," Mathematical and Computer Modelling, vol. 54, no. 9, pp. 2544-2550, 2011.

[21] W. F. Al-Omeri, O. H. Khalil, and A. Ghareeb, "Degree of $(L, M)$-fuzzy semi-precontinuous and $(L, M)$-fuzzy semi-preirresolute functions," Demonstratio Mathematica, vol. 51, no. 1, pp. 2391-4661, 2018.

[22] A. Ghareeb and F.-G. Shi, "SP-compactness and SP-connectedness degree in $L$-fuzzy pretopological spaces," Journal of Intelligent \& Fuzzy Systems, vol. 31, no. 3, pp. 1435-1445, 2016.

[23] A. Ghareeb and W. F. Al-Omeri, "New degrees for functions in $L, M$-fuzzy topological spaces based on $L, M$-fuzzy semiopen and $L, M$-fuzzy preopen operators," Journal of Intelligent and Fuzzy Systems, vol. 36, no. 1, pp. 787-803, 2019.

[24] A. Ghareeb, "Degree of $F$-irresolute function in $L, M$-fuzzy topological spaces," Iranian Journal of Fuzzy Systems, vol. 16, no. 4, pp. 189-202, 2019.

[25] A. Ghareeb, H. S. Al-Saadi, and O. H. Khalil, "A new representation of $\alpha$-openness, $\alpha$-continuity, $\alpha$-irresoluteness, and $\alpha$-compactness in L-fuzzy pretopological spaces," Open Mathematics, vol. 17, no. 1, pp. 559-574, 2019.

[26] H. Li and Q. Li, "RL-topology and the related compactness," Journal of Mathematical Research with Applications, vol. 38, no. 6, pp. 636-642, 2019.

[27] H. Li and Q. Li, " $R L$-Fuzzy topology and the related fuzzy compactness," Journal of Shandong University (Natural Science), vol. 54, no. 2, pp. 51-57, 2019, in Chinese.

[28] W. Guo-Jun, "Theory of topological molecular lattices," Fuzzy Sets and Systems, vol. 47, no. 3, pp. 351-376, 1992.

[29] U. Höhle and A. P. Šostak, Axiomatic Foundations of Fixed-Basis Fuzzy Topology, Springer, Boston, MA, USA, 1999.

[30] S. E. Rodabaugh, Categorical Foundations of Variable-Basis Fuzzy Topology, Springer, Boston, MA, USA, 1999. 